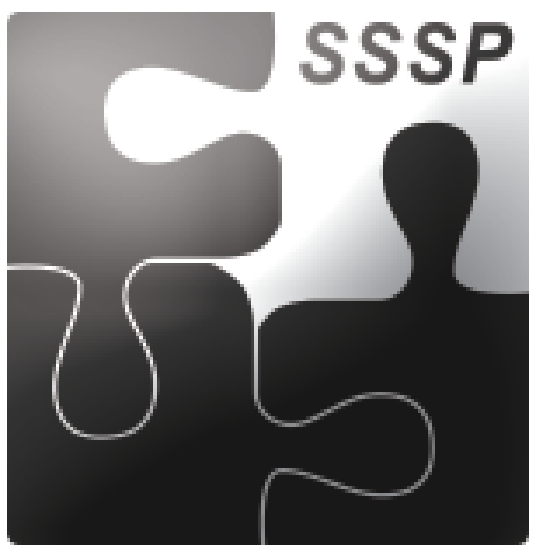

Acculturation and Self-Rated Health among Latino and Asian Immigrants to the United States Author(s): Rachel Tolbert Kimbro, Bridget K. Gorman and Ariela Schachter

Source: Social Problems, Vol. 59, No. 3 (August 2012), pp. 341-363

Published by: University of California Press on behalf of the Society for the Study of Social Problems

Stable URL: http://www.jstor.org/stable/10.1525/sp.2012.59.3.341

Accessed: 03/07/2013 16:52

Your use of the JSTOR archive indicates your acceptance of the Terms \& Conditions of Use, available at

http://www.jstor.org/page/info/about/policies/terms.jsp

JSTOR is a not-for-profit service that helps scholars, researchers, and students discover, use, and build upon a wide range of content in a trusted digital archive. We use information technology and tools to increase productivity and facilitate new forms of scholarship. For more information about JSTOR, please contact support@jstor.org. 


\title{
Acculturation and Self-Rated Health among Latino and Asian Immigrants to the United States
}

\author{
Rachel Tolbert Kimbro, Rice University
}

Bridget K. Gorman, Rice University

Ariela Schachter, Stanford University

\begin{abstract}
The ways in which immigrant health profiles change with shifts in acculturation is of increasing interest to scholars and policy makers in the United States, but little is known about the mechanisms that may link acculturation and self-rated health, particularly for Asians. Utilizing the National Latino and Asian American Study (NLAAS) and its data on foreign-born Latinos $(\mathrm{N}=1,199)$ and Asians $(\mathrm{N}=1,323)$ (Pennell et al. 2004), we investigate and compare the associations between acculturation and self-rated health for immigrants to the United States from six major ethnic subgroups (Chinese, Filipino, Vietnamese, Mexican, Cuban, and Puerto Rican). Using comprehensive measures of acculturation, we demonstrate that across ethnic groups, and despite the widely varying contexts of the sending countries and receiving communities, native-language dominance is associated with worse self-rated health relative to bilingualism, and measures of lower acculturation-coethnic ties and remittances-are associated with better self-rated health; and moreover, these associations are only partially mediated by socioeconomic status, and not mediated by acculturative stress, discrimination, social support, or health behaviors. We speculate that immigrants who maintain a native language while also acquiring English, as has been shown for other immigrant outcomes, attain a bicultural fluency, which also enables good health. Surprisingly, we do not find strong associations between duration of time in the United States or age at migration-measures frequently used to proxy acculturation-with self-rated health. Our findings illustrate the complexity of measuring acculturation and its influence on health for immigrants. Keywords: acculturation; migration; health; language; ethnicity.
\end{abstract}

In the United States today, approximately one in five persons resides in an immigrant familybeing either foreign born or the child of immigrants (Hirschman and Massey 2008). A large proportion of population growth over the past 20 years in urban and nonurban areas of the United States is attributable to births in these immigrant families (Johnson and Lichter 2008), although immigrants, while still concentrated in several states and large urban areas, are residing more often in new destinations (Singer 2004). At the same time, a rise in American antipathy towards immigrants, coupled with spiraling health care costs and a new attempt at health care reform, have created a great need for reliable data on the health and well-being of immigrant groups, and how their incorporation into the United States may influence that health and well-being. Moreover, to date, research on immigrant incorporation has tended to utilize unidimensional measures of acculturation (such as the number of years lived in the United States), despite there being strong theoretical and empirical arguments for complexity underlying the acculturation process, highlighting the need for multidimensional and ecodevelopmental frameworks relating acculturation to health (Lopez-Class, Castro, and Ramirez 2011).

Direct correspondence to: Rachel Tolbert Kimbro, Department of Sociology, MS-28, Rice University, 6100 Main St. Houston, TX 77005; E-mail: rtkimbro@rice.edu.

Social Problems, Vol. 59, Issue 3, pp. 341-363, ISSN 0037-7791, electronic ISSN 1533-8533. ( ) 2012 by Society for the Study of Social Problems, Inc. All rights reserved. Please direct all requests for permission to photocopy or reproduce article content through the University of California Press's Rights and Permissions website at www.ucpressjournals.com/reprintinfo/asp. DOI: 10.1525/sp.2012.59.3.341. 
Focusing on the health status of immigrants, which tends to erode over time spent in the United States-and with acculturation-provides a good case study for the acculturation process, and highlights the extent to which continuing migration into the United States may shape not only the overall health profile of the U.S. population, but the level of need for health-related resources and expenditures in the years to come.

Approximately 80 percent of the foreign born in the United States are from Asian or Hispanic subgroups (Grieco 2010; Larsen 2004). Overall, studies have shown that immigrants come to the United States in better health than their native-born counterparts, but that this benefit erodes with time (Antecol and Bedard 2006; Cho and Hummer 2001; Singh and Siahpush 2002). This "healthy immigrant effect" is all the more surprising given that the socioeconomic profile of many immigrant groups is quite low, and research has repeatedly linked poorer health outcomes to lower socioeconomic status (SES) (House and Williams 2000). Research on self-rated health has further complicated our understanding of immigrant health status, as some studies find better selfrated health among recent immigrants, while others find the reverse (Zsembik and Fennell 2005), a pattern surely complicated by research indicating that the understanding of the actual self-rated health question by immigrant groups may differ from the native born, due to language and interpretation issues (Angel and Guarnaccia 1989; Bzostek, Goldman, and Pebley 2007). Therefore, a need exists for research that both compares self-rated health across immigrant groups, and attempts to disentangle relationships among acculturation, language, and self-rated health (Lopez-Class et al. 2011).

In this article, we utilize a nationally representative U.S. data set that includes large samples of Latino and Asian foreign-born adults to test how acculturation is related to self-rated health across six major subgroups (Chinese, Filipino, and Vietnamese ethnic groups among the Asian American sample, and Mexican, Cuban, and Puerto Rican ethnic groups among the Latino sample). We measure acculturation in comprehensive and unique ways, focusing on language ability in both the respondent's language of origin and English, the frequency of remittances and return visits, age at migration, the closeness of coethnic ties, and whether he/she migrated to find a job. We also explore socioeconomic status, stress and discrimination, social support, and health behaviors as explanatory mechanisms for the relationships between acculturation and self-rated health. Finally, we test the correlations between self-rated health and several objective health measures for each ethnic group to assess whether the meaning of the self-rated health question is likely to vary across groups.

\section{Background}

\section{Acculturation and Self-Rated Physical Health}

Self-rated health is increasingly acknowledged to be an important indicator of health status. It is a valid predictor of mortality, even after controlling for a variety of objective health measures (Idler and Benyamini 1997), and studies have repeatedly shown that differences in self-rated health across population subgroups mimic differences seen in more objective measures of health status (Idler, Rusell, and Davis 2000; Månsson and Råstam 2001) and mortality (Franks, Gold, and Fiscella 2003; Idler and Angel 1990). Therefore, it is an expedient measure of the general health status of individuals that maps well onto differences in more objective health measures. In addition, we argue that if broad health differences across groups are of interest, a subjective, holistic measure like self-rated health captures the totality of an individual's health status in a way that a single objective measure (or a scale of multiple objective measures) could not. In terms of racial/ethnic health disparities in the measure, studies have found that black adults are most likely to report fair or poor self-rated health, followed by Hispanics, then non-Hispanic whites, and finally Asians (McGee et al. 1999; Meredith and Siu 1995), although more recently Namratha Kandula, Diane Lauderdale, and David Baker (2007) found Asians to have worse self-rated health compared 
to whites. To date, most health disparities research has focused on the black-white divide in self-rated health, while less is known about Latinos and particularly Asians.

Researchers often focus on acculturation as a possible explanation for self-rated health patterns among Latino and Asian adults. While exact definitions vary, acculturation is generally understood to be the process by which immigrants are exposed to the receiving country's culture and, over time, adapt characteristics of this culture while simultaneously rejecting characteristics of the culture of their country of origin (Alba and Nee 1997). Of course, the large social and political differences in both countries of origin as well as in the contexts of receiving communities (Menjívar 2000; Portes and Rumbaut 2001; Portes and Zhou 1993) imply that the relationships between acculturation and health most likely will vary for each immigrant subgroup. In healthrelated research, most studies test the general hypothesis that increasing acculturation decreases differences between whites and Latinos or Asians on rates of fair/poor self-rated health (AbraídoLanza, Chao, and Florez 2005). For example, Susan Shetterly and colleagues (1996) found that even controlling for SES, acculturation was a significant predictor of low self-rated health among Latinos, and that the most acculturated Latinos had the most similar self-rated health when compared to whites. Other studies have found similar results for Asian immigrants (e.g., Frisbie, Cho and Hummer 2001).

With regard to Latinos, some studies have shown that the less acculturated report worse selfrated health (Franzini and Fernandez-Esquer 2004), findings that seem counterintuitive given the widely documented healthy immigrant effect found using more objective health status measures. However, Brian Finch and William Vega (2003) found that, controlling for acculturation status, Mexican immigrants reporting higher levels of acculturative stress report poorer self-rated health, suggesting that it is not necessarily those who are most or least acculturated who report the poorest self-rated health, but rather those most affected by the stress associated with the acculturation process. Sharon Bzostek, Noreen Goldman, and Anne Pebley (2007) offer an alternative explanation, suggesting that there may be two distinct processes occurring simultaneously: (1) increasing acculturation leads Latinos to rate their health more positively, and (2) the adoption of negative health behaviors more prevalent in U.S. society results in Latinos rating their health worse, and these two processes counteract and effectively cancel out one another. With regard to Asians, much less attention has been paid to the relationship between self-rated health and acculturation, and a need exists for documenting potential differences across Asian ethnic groups. While Elena Erosheva, Emily Walton, and David Takeuchi (2007) found no difference in self-rated health when comparing foreign and U.S.-born Asians, Parker Frisbie, Youngtae Cho, and Robert Hummer (2001) found that Asian immigrants reported better self-rated health compared to U.S.-born Asians, but that their health advantage decreased over time. Most recently, Zhang and Ta (2009) also found that compared to U.S.-born Asians, recent immigrants were less likely to report low self-rated health.

\section{Measuring Acculturation}

Most research concerning linkages between acculturation and self-rated health focuses on how time in the United States shifts the cultural and social orientation of immigrants from their home country to the United States, and is thought to encompass a variety of aspects of life (e.g., language, food, social integration, access to and utilization of health care). Many health disparities studies define and test acculturation as a unidimensional transition from sender-country culture to receiver-country culture (e.g., Palinkas and Pickwell 1995; Salant and Lauderdale 2003), most commonly measured using nativity (U.S. or foreign born) and length-of-stay in the United States (e.g., Erosheva et al. 2007). More sophisticated analyses also examine language proficiency and usage, including measures of how much respondents think, write, and use media in English and Spanish for Latinos (Finch and Vega 2003; Franzini and Fernandez-Esquer 2004; Schachter, Kimbro, and Gorman 2012). We found similar acculturation measures for nativity and length-ofstay for Asians, but fewer studies were able to use English proficiency because few U.S.-born 
respondents did not speak English (Erosheva et al. 2007). However, according to a recent review by Maria Lopez-Class, Felipe González, and Amelie Ramirez (2011), this common reliance on indicators of language usage and generation status does not capture the nuances involved in the acculturation-health relationship.

In contrast to this unidimensional focus, others have argued for a bidimensional model of acculturation (An, Cochran, and Mays 2008; Rogler, Cortes and Malgady 1991; Ryder, Alden, and Paulhus 2000), wherein adaptation to the host country and maintenance of country-of-origin practices are considered independently (Berry 1997). Each of these categories of acculturation may have different effects on health (Bethel and Schenker 2005), but we expect that in general, evidence of maintaining ties to the sending country, in conjunction with evidence of acculturating to the United States, is likely to be beneficial for the health of immigrants. In line with this perspective, scholars examining language usage have argued that it, too, should not be seen as a linear, unidimensional transition from Spanish to English (for Latinos), as some Latinos with high English-proficiency may maintain their Spanish language skills (Hunt, Schneider, and Comer 2004). However, studies focused on Asians have noted that for some subethnic Asian groups (e.g., Filipinos and selected South Asians), English is the native language, limiting its usage as a measure in analyses among Asian subethnic groups (Salant and Lauderdale 2003). Nonetheless, we expect language proficiency to be a key predictor of self-rated health for immigrants, since immigrants who are bilingual-highly proficient in both English and their native language-also can be expected to have a high degree of "cultural flexibility" that expands their social networks and increases their knowledge of and access to healthy behaviors and health care (Chen, BenetMartínez, and Bond 2008). In prior work testing the associations between language proficiency and physical and mental health for panethnic Asian and Latino groups, we found that being bilingual was associated with better health compared to being native-language or English dominant (Schachter et al. 2012). Thus, we also hypothesize that simply being English proficient—while having reduced proficiency in a native language-is unlikely to confer the same benefits to health, because it indicates a loss of familiarity with one's sending country and a lower likelihood of being connected to two cultures.

Scholarship examining the health effects of acculturation tends to rely on measures of adaptation to U.S. society (e.g., (Finch and Vega 2003; Franzini and Fernandez-Esquer 2004), but rarely do these studies also examine the extent to which immigrants maintain country-of-origin ties and healthy behaviors. Yet, it seems likely that immigrants would benefit from close connections to country-of-origin family and social networks, as these connections are thought to encourage healthy behaviors and social supports that promote physical and mental well-being (Kimbro 2009). For example, previous research, mostly focused on Latino immigrants, has found that foreign-born residents of the United States who remit money to relatives in their respective countries-of-origin tend to be more recent migrants with the strongest personal links back home (DeSipio 2002; Lozano-Ascencio 2005; Sana and Massey 2005). Given this evidence, we would expect that remitting would be a sign of links to the sending country, and, following previous findings of the healthy immigrant effect, we hypothesize that remitting individuals would be likely to have higher levels of self-rated health compared to individuals who do not remit.

In addition to considering social ties to the country of origin, we also examine immigrants' self-reported levels of ethnic identity and closeness to others of the same race/ethnicity as an additional aspect of bidimensional acculturation. Here we are informed by Jean Phinney's (1990) definition of ethnic identity as a measure of respondents' own sense of belonging to a culture or group. Previous scholarship has found that a strong ethnic identity can protect health status by shielding immigrants from negative health behaviors and by increasing the monitoring of health status and health behaviors by coethnic group members (Phinney 1990; Phinney et al. 2001; Frisbie et al. 2001). Additionally, Krysia Mossakowski (2003) argued that ethnic identity can be an important source of social support and that it is protective for immigrant mental health status. We correspondingly hypothesize that immigrants with a stronger sense of closeness to coethnics will report better self-rated health relative to those who feel less close. 
Following a similar line of reasoning, John Berry (1997) and Talya Salant and Diane Lauderdale (2003) have argued that additional contextual factors such as reason for migrating (e.g., economic opportunities) can frame how acculturation occurs for individuals. This context for migration may be particularly important in health research given the selection argument - the healthy immigrant effect may be explained by the necessity of good health in order to make the often arduous and physically demanding trip to the United States, as well as the high prevalence of young, employmentseeking males (who tend to have few health problems) among migrators (Jasso et al. 2004; Landale, Oropesa, and Gorman 2000). In particular, economic migrants-those whose primary motive for migration is to find a job-may experience more selection effects relative to other immigrants (including those whose migration is primarily motivated by family reunification or political asylum) based not just on health but also other indicators of labor market success (Chiswick 1999), Together, this scholarship suggests that economically motivated migrants will report better health statuses compared to other immigrants. Another way to measure immigrant country-of-origin ties is the frequency of return trips they make. Douglas Massey and Ilana Akresh (2006) have emphasized that not all immigrants come to the United States with the intention to remain permanently and become naturalized citizens. The permanence of migration depends on many factors, including the economic and social conditions in sending nations, and how satisfied migrants are with their lives in the United States. Frequency of return trips may tap an individual's intended degree of permanence in the United States, and thus acculturation level.

\section{Explanatory Mechanisms}

The relationship between acculturation and self-rated health is almost certainly related to socioeconomic status, and this relationship likely differs across immigrant subgroups due to the varied contexts of sending countries and receiving communities, as well as different modes of emigration (Lara et al. 2005). For example, some Asian immigrants to the United States are highly educated, enjoy good access to health care, and have high levels of English ability (Brown et al. 2000). In contrast, many immigrants from Mexico have low levels of education and economic status in the United States (Markides and Eschbach 2005), although this is not necessarily the case across all Latino ethnic groups. For example, Cuban immigrants are likely to be highly educated, enjoy relatively high socioeconomic status, and have better health outcomes compared to other Latinos (Vega and Amaro 1994). We expect that the relationship between acculturation and health will be partially explained by SES; that is, that education and income-related measures are responsible for some of the relationship between acculturation and health. For example, bilingual immigrants might report better health partially because they are also more likely to be better educated. Or, immigrants who make more frequent return trips to their countries of origin might report better health because they are also likely to have higher incomes. Research also demonstrates, however, that the relationship between SES and health and health behaviors is unusual for U.S. immigrant groups (Goldman et al. 2006; Kimbro et al. 2008), and that these "health gradients" are typically flatter for immigrants than for the U.S. born, indicating a reduced salience of SES factors for predicting immigrant health. If this is the case, we may not see much mediation of acculturation measures by SES measures. Measures of acculturation are also likely bound together with SES in ways that might shape physical health outcomes. For example, research on remittances has shown a positive relationship between SES and remitting (Lozano-Ascencio 2005). However, Massey and Akresh (2006) have argued that attachment to the United Statesand thus one's likelihood of remitting-is not inherently determined by SES, emphasizing that highly educated immigrants who earn high incomes are positioned to be very desirable workers within the global labor market, and therefore may face little pressure to assimilate. It is unclear how remittances may be tied to self-rated health, but we suspect that more remitting behavior will be linked to better health, and that this may be partially explained by the higher SES necessary for remitting. Alternatively, more remitting might be an indicator of needy family members who have been left behind in the country of origin, and therefore a source of stress that is linked to 
poorer health, particularly for immigrants with lower SES (Ward 2010). We thus hypothesize that SES will be a key mediator of the relationships among our acculturation measures and self-rated health.

We hypothesize further that stress, discrimination, social support, and health behaviors are additional, but likely less consequential, mediators of the relationship between acculturation and self-rated health. As much as the migration-health scholarship emphasizes the healthy selection of migrants (e.g., Jasso et al. 2004), this does not overshadow the difficulties associated with moving from one country to another, particularly the upheaval of relationships with friends and family, as migrants break ties with those left behind and seek out new relationships and sources of support in the United States (Rogler 1994). Previous scholarship has demonstrated that both recent migrators and more acculturated immigrants can experience high levels of stress relating to resettlement (Kritz and Gurak 2004; Krueger and Chang 2008) and perceived discrimination (Williams, Neighbors, and Jackson 2008), which may impact their physical health status (Finch and Vega 2003). However, scholars note that the migration process also relies on, and reinforces, strong social network support, as migrants often settle in areas where they know people and find other coethnic residents (Arnold 1989; Martin and Midgeley 2006; Sanders, Nee, and Sernan 2002; Vega et al. 1991). As such, strong social and family support may counteract the effects of stress, discourage unhealthy behaviors, and provide positive role models (Finch and Vega 2003; Kawachi and Berkman 2000; Thomson and Hoffman-Goetz 2009). The strength of family and social ties may depend on individuals' levels of acculturation into larger communities (Alba and Nee 2003); however, Bridget Gorman, Elaine Ecklund, and Holly Heard (2010) find that measures of integration and social support mediate little of the relationship between immigrant status and physical health. In the analysis that follows, we test associations between acculturation and self-rated health for six major U.S. immigrant groups, and assess whether the relationship between acculturation and self-rated health are driven by SES or other explanatory factors.

\section{Data, Measures, and Method}

\section{Data}

We examine data on foreign-born adults from the National Latino and Asian American Study (NLAAS). Collected in 2002-2003, the NLAAS is a nationally representative community household survey designed to examine mental health and health care among U.S. Latinos and Asians Americans aged 18 and older. A multistage, stratified national area probability sample was drawn from the noninstitutionalized U.S. population, with oversampling of areas with a moderate-tohigh density of Latinos and Asian Americans. All interviewers were bilingual, and interviews were conducted in person and in English, Spanish, Vietnamese, Chinese (either Mandarin or Cantonese), or Tagalog. The overall response rate was 65.6 percent for Asian Americans and 75.5 percent for Latinos (see Heeringa et al. 2004 and Pennell et al. 2004 for detailed sampling descriptions). When weighted, the NLAAS includes a nationally representative sample of 4,649 adults, including 2,554 Latinos (including Mexican, Puerto Rican, Cuban, and other Latino groups) and 2,095 Asian Americans (including Chinese, Vietnamese, Filipino, and other Asian groups). We limit this sample based on three criteria. First, because of our focus on acculturation, we limit the sample to foreign-born respondents, or immigrants $(n=3,266)$. Next, given our interest in ethnic group differences, we exclude respondents who do not identify with a specific ethnic group (i.e., "other" Latino and Asian Americans; $n=738$ ). Last, we remove one case with missing information on self-rated health, resulting in a final sample size of 2,522 respondents. Rates of item nonresponse are nonexistent or small (under 2 to 3 percent for most measures), with the exception of one measure, index of neighborhood trust (see measures sections), where 15 percent of cases are missing. All missing data on predictor measures were imputed using the multiple imputation ICE command in Stata. 


\section{Measures}

Our dependent measure is self-rated health, where respondents are asked to rate their overall physical health status on a five-point scale $(1=$ poor and $5=$ excellent $)$. Our key predictors are several measures of acculturation that tap different but interrelated aspects of the acculturative process for U.S. migrants. This includes indicators of age at migration to the United States $(1=$ before age 18, $0=$ age 18+); whether they currently remit money to relatives in their country of origin $(1=$ yes, $0=$ no); how frequently they make return visits to their country of origin $(1=$ never, $2=$ rarely, $3=$ sometimes, and $4=$ often); and the importance of finding a job as their reason for migrating to the United States ( $1=$ not at all important, 2 = somewhat, and 3 = very important). We also examine a measure of coethnic ties, based on the average response to four questions that ask respondents to rank how close they feel to others of the same racial/ethnic descent (e.g., "How closely do you identify with other people who are of the same racial and ethnic descent as yourself?"; $\alpha=.66$ ). Finally, we include a measure of whether the respondent is a U.S. citizen ( $1=$ citizen). Given the multidimensional qualities of the acculturation process, compared to creating an acculturation scale, including each of these measures allows for maximum flexibility in measurementrespondents can be high on one measure and low on another. None of the measures were correlated more than $r=.23$.

The NLAAS also includes measures of language use and ability, and we measure both English and native language proficiency, where respondents are asked to rate their ability to read, write, and speak English and Asian/Spanish on four-point scales $(1=$ poor and $4=$ excellent). Respondents are also asked how much they use each language on a five-point Likert scale ( 1 = only Asian/Spanish and $5=$ only English) in a variety of contexts, including with family, with friends, and when thinking. Additionally, the NLAAS includes interviewer-rated scores of English proficiency for respondents who took the survey in English. John Ayers (2010) has argued that interviewer-assessed measures of language ability may be more accurate than self-assessed ratings, and using NLAAS data found significant differences in self- and interviewer-assessed ratings for individual respondents. However, the NLAAS does not include interviewer-assessed measures of fluency in native languages. This means that the interviewer-assessed English fluency ratings promoted by Ayers (2010) are not appropriate for our analysis given our specific interest in bilingualism. Also, bilingual English-Spanish speakers were randomly assigned to either the English or Spanish version of the survey, while all other bilingual respondents were allowed to choose which language they preferred for the interview, meaning that interviewer-assessments of English fluency for some bilingual speakers are not included. As such, we cannot consider language of interview alone to be an accurate measure of English fluency, as some bilingual Latinos were randomly assigned to the Spanish survey instrument. Nevertheless, we recognize Ayers' (2010) and Akresh and Reanne Frank's (2011) caution of using self-reported language proficiency, and note the potential for bias in these measures.

We calculated Pearson correlation coefficients for all combinations of the self-rated English and native-language variables for reading, writing, and speaking, along with all of the context of use language measures, and based on the high multicollinearity present among these variables we decided to create scaled measures that would allow us to include multiple indicators of language use and context without introducing instability into our models. First, English proficiency is calculated by summing the three measures of respondents' abilities to read, write, and speak English $(\alpha=$.97). Higher values on the scale indicate greater proficiency in English. Second, Asian/Spanish (native language) proficiency is based on three measures and is the sum of respondents' values for how well they read, write, and speak their native language $(\alpha=.94)$. Next, we used cluster analysis (k-means) to discern a sensible grouping of respondents into categories so we could model proficiency in both languages simultaneously, which resulted in: bilingual (high proficiency on both scales), native-language dominant (high on native language proficiency but not English), and English dominant (high on English proficiency but not on native language). Next, the three measures for the frequency of English use among friends, family, and while thinking were summed 
into a scale $(\alpha=.86)$ and divided by three for an average score. Thus, this measure gives the average propensity to use English in several different contexts, and allows us to test whether language proficiency still matters even in the context of accounting for the frequency of use of English.

We also examine several categories of covariates. Demographic characteristics include gender ( 1 = female) and age at interview (range: 18 to 97). We include seven key measures of socioeconomic status, including dummy variables for completed schooling, which contrasts adults who (1) did not complete high school against (2) those who graduated from high school, and (3) adults with at least some college experience. We also include dichotomous measures of employment ( 1 = currently working, $0=$ otherwise) and poverty status ( $1=$ income below the 2001 federal poverty line, 0 = higher), while for relative income respondents rank from 0 to 10 how well off they are relative to all other people in the United States (where higher values $=$ more well off). We also include measures of the extent to which respondents report that they don't have enough money to meet their needs (where 1 = more than enough, 2 = just enough, and $3=$ not enough), and whether adults lack any type of medical insurance coverage.

Several measures of stress and discrimination are also available in NLAAS, including acculturative stress, which is a summed index $(\alpha=.71)$ based on responses to nine yes-no questions about stress experienced since migrating to the United States (e.g., "Have you felt guilty about leaving family or friends in your country of origin?"). We also include the frequency of day-to-day discriminatory treatment on the basis of national origin/ancestry, race, or skin color (where $1=$ never and $6=$ almost everyday), constructed from the average of nine questions about routine experiences with racial discrimination (e.g., being treated with less respect than other people, being called names or insulted; $\alpha=.91$ ). We also construct two measures that tap negative aspects of relationships with friends and family. First, our measure of negative social exchanges is an averaged index based on four questions that ask how frequently friends and family argue with and make too many demands on the respondent (where $1=$ less than once a month and $5=$ almost every day; $\alpha=.69)$. Second, family cultural conflict is an averaged index $(\alpha=.76)$ based on five questions addressing issues of cultural and intergenerational conflict between respondents and their families (e.g., arguments over different customs, personal goals in conflict with family), ( 1 = hardly ever or never, 2 = sometimes, and 3 = often) (see Alegria et al. 2004).

We also control for multiple measures of social networks and support, including marital status ( $1=$ married or cohabiting, $0=$ otherwise) and the number of adults and children living in the household. Additionally, we construct an index of neighborhood trust, which is the average of seven questions $(\alpha=.81)$ about perceptions of neighborhood safety and social cohesion (e.g., how safe the respondent feels being out alone in his or her neighborhood at night, whether people in the neighborhood can be trusted), where $1=$ not at all true and $4=$ very true. Positive social support is constructed from six questions $(\alpha=.73)$ that gauge the availability of support from friends and family (e.g., how much they can rely on relatives they don't live with if they have a serious problem), where $1=$ less than once a month and $5=$ almost every day. Family cohesion is constructed from 10 questions $(\alpha=.93)$ that gauge family closeness and communication (e.g., family members like to spend free time with each other, family members feel very close to each other) ( 1 = hardly ever or never, 2 = sometimes, and 3 = often).

Finally, we control for three health behaviors, including three dummy variables for smoking status (current smoker, former smoker, and never smoked), while heavy drinking is defined as two or more drinks per day for women, and three or more drinks per day for men (USDHHS 2005). Last, we measure whether respondents are classified as obese $(1=$ yes, $0=$ no), based on their body mass index (WHO 1995).

\section{Method of Analysis}

All analyses were run using the Stata 12.0 software package. We utilized Taylor-seriesapproximate methods with SVY commands to adjust for the complex sample design of the NLAAS. All analyses in this article are also weighted with the final sampling weight. We begin by 
presenting sample characteristics for foreign-born NLAAS adults, followed by OLS regression models predicting self-rated physical health for both Latino and Asian American adults, focusing on the role of acculturation in predicting self-rated health, and how this differs across Latino and Asian ethnic groups. We ran our modeling sequence for self-rated health using both ordered logit and OLS regression models, as well as logistic models predicting both good/excellent self-rated health and fair/poor self-rated health; findings were very similar, so we only present results from OLS models.

\section{Results}

\section{Sample Characteristics}

Weighted NLAAS sample characteristics and bivariate tests for difference, by ethnic subgroup, are displayed in Table 1. First, note that Filipinos and Cubans have the highest mean score on self-rated health for Asians and Latinos, respectively. In terms of the acculturation measures, while the vast majority (over three-quarters) of Asian subgroups migrated after age 18, we see differences for the Latino subgroups-more Cubans came to the mainland United States at older ages compared to Mexicans and Puerto Ricans. We also see important differences across groups in our language measures. For Asians, compared to the Chinese subgroup (38.2 percent), more Filipinos are bilingual (64.5 percent), likely due to the widespread use of English in the Philippines. In addition, also compared to those of Chinese origin (14.7 percent), significantly fewer Vietnamese immigrants in the United States report high English ability (8.1 percent). For the Latino subgroups, fewer Cubans and Puerto Ricans are Spanish-dominant (61.2 percent and 42.4 percent, respectively) compared to Mexicans (79.4 percent).

Compared to immigrants of Chinese origin, those from Vietnam return less often. Compared to those of Mexican origin, those from Cuba return less often, and those from Puerto Rico return more often, likely due to the legal barriers preventing travel between Cuba and the United States and the ease of traveling between the United States and Puerto Rico. Remittances also show different patterns across the subgroups. Filipinos and Vietnamese remit at nearly twice the rate of Chinese, while Puerto Ricans are much less likely to do so than either Mexicans or Cubans. Filipinos are more likely than Chinese immigrants to cite the importance of finding work for their migration decision, while compared to Mexican immigrants, Cubans and Puerto Ricans are less likely to cite a job search as a reason for migrating. Filipinos and Vietnamese immigrants report stronger coethnic ties compared to Chinese immigrants, and Cubans report stronger coethnic ties compared to Mexicans. Finally, Vietnamese immigrants are more likely than are Chinese immigrants to report being a citizen, while Cubans are significantly more likely than Mexicans to report being a citizen.

The groups also differ widely, as expected, by socioeconomic status. More than half of the Chinese and Filipino immigrants in our sample have at least some college education, compared to percent for the Vietnamese immigrants and just 12.8 percent for Mexican immigrants, 40.3 percent for Cubans, and 33.3 percent for Puerto Ricans. Vietnamese, Cuban, and Puerto Rican immigrants also demonstrate a bimodal educational distribution-with large proportions at the low end (less than high school) and high end (some college or more). This illustrates the heterogeneity within immigrant subgroups, and not just between them. With the exception of Filipinos, all immigrant groups have close to or more than one-third of respondents living in poverty.

\section{Additive Regression Models}

Tables 2 and 3 present results of our first series of OLS regression models predicting self-rated health for all six of our immigrant subgroups. Table 2 presents results for Chinese, Filipino, and Vietnamese immigrants, while Table 3 presents results for Mexicans, Cubans, and Puerto Ricans. We show a series of three stepwise models for each group, starting with our baseline models that 


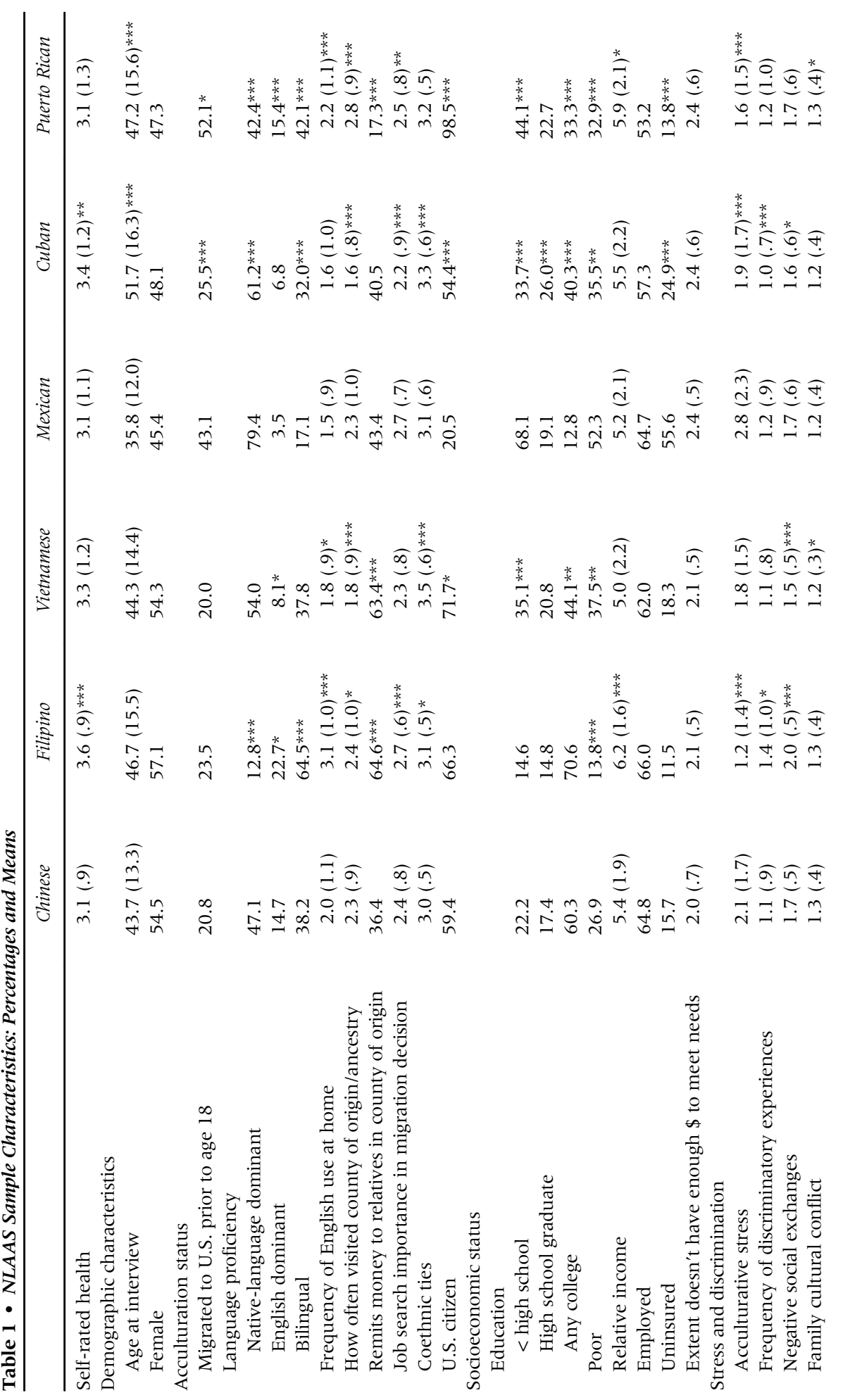




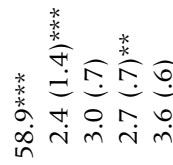

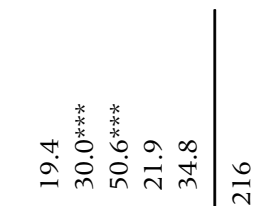

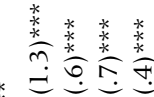

in

$\stackrel{\infty}{=} \widehat{0} \widehat{\underline{n}}$

ن.

$n$

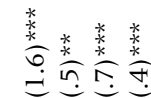

$\infty$ in $\begin{aligned} & \text { 4 } \\ & 0\end{aligned}$

ஸि थि में

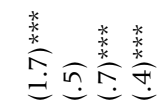

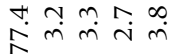

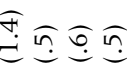

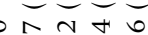

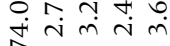

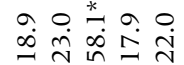

$\sqrt{7}$

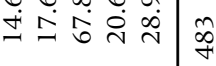

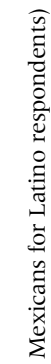

究

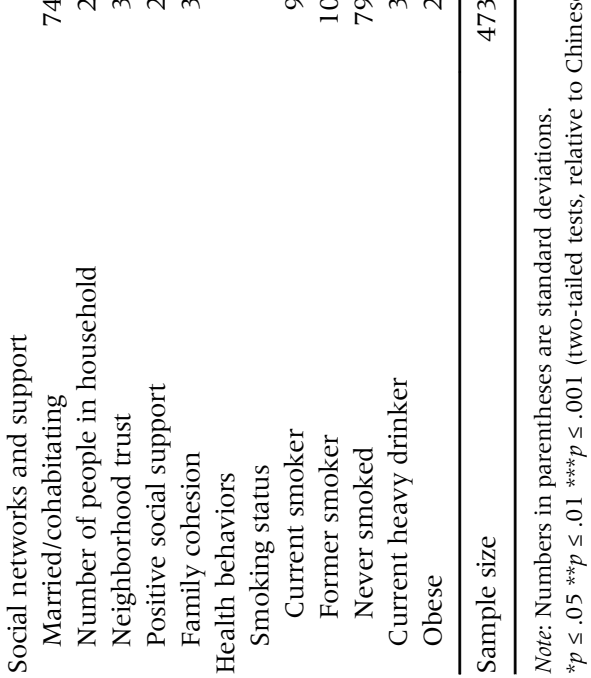




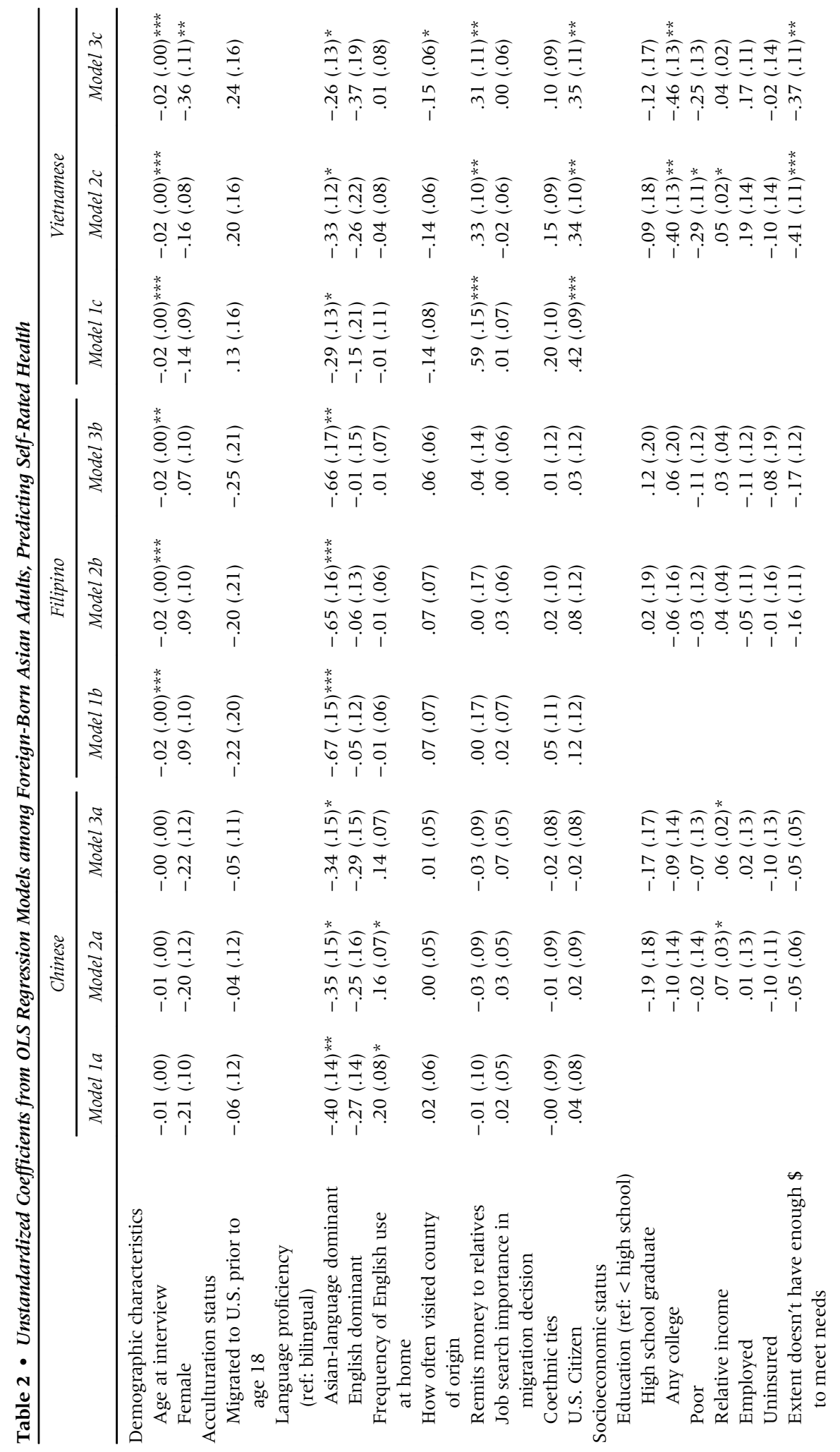




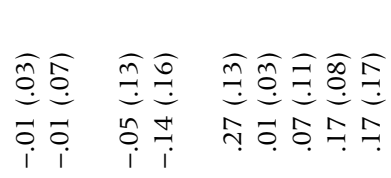

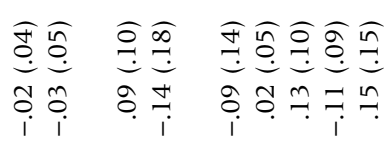

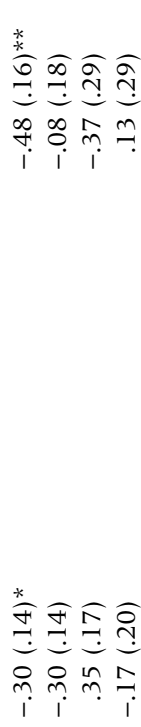

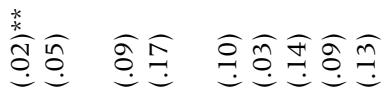

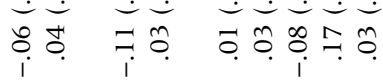

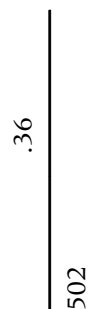

in

กั.

(1)

ก

市

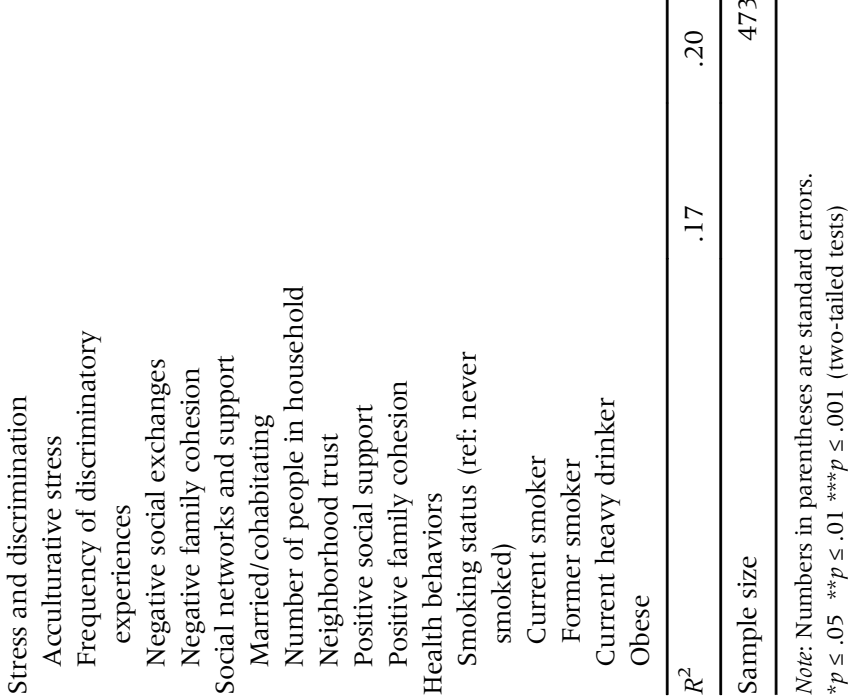

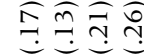

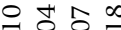

$\stackrel{n}{\Upsilon}$

$\because$

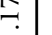

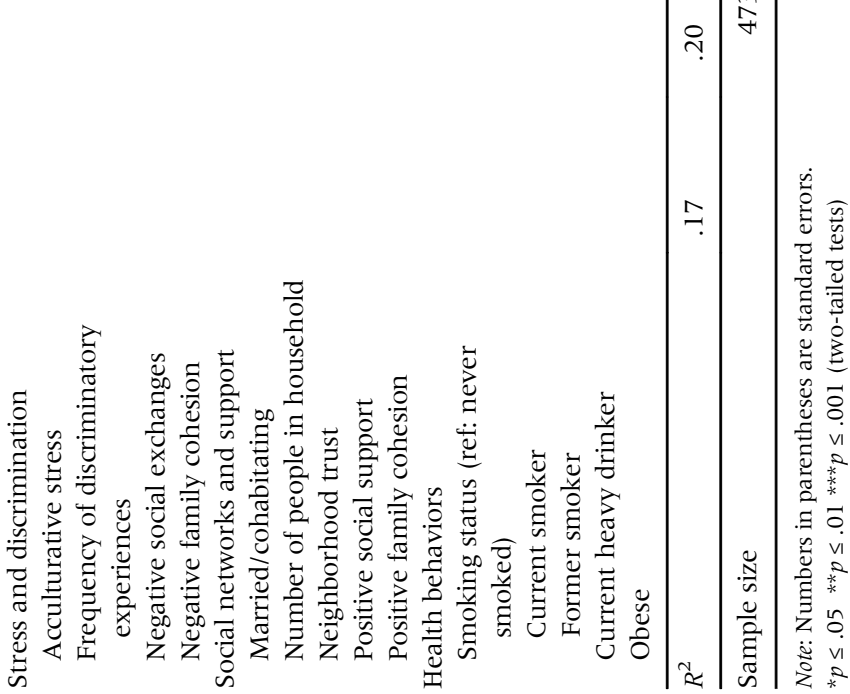




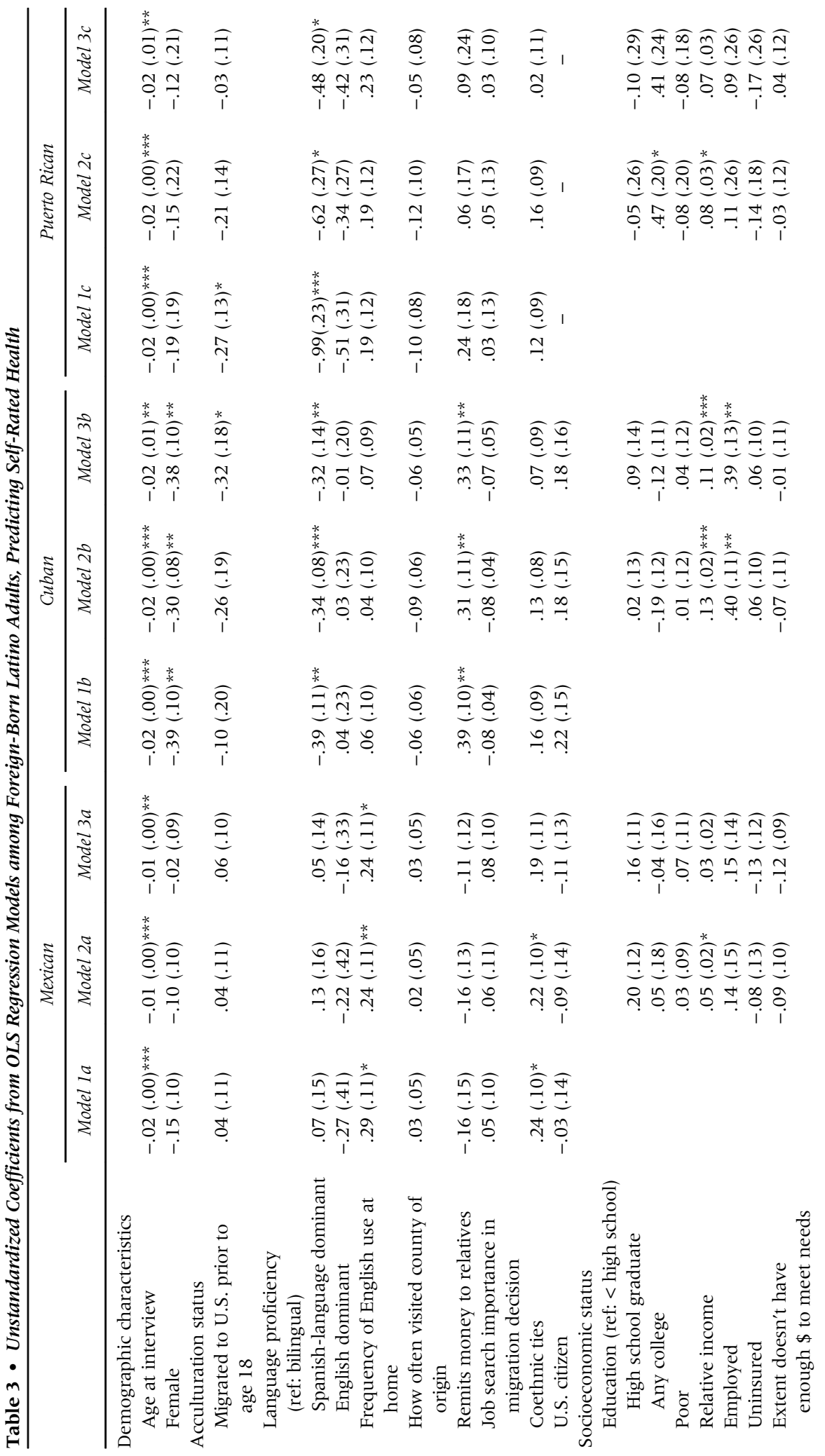




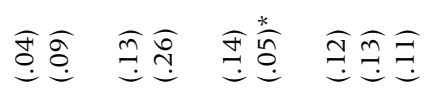

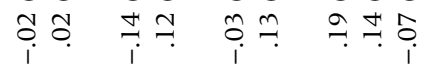

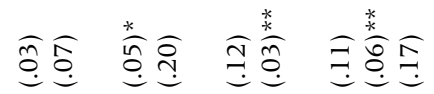

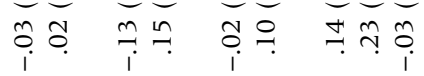

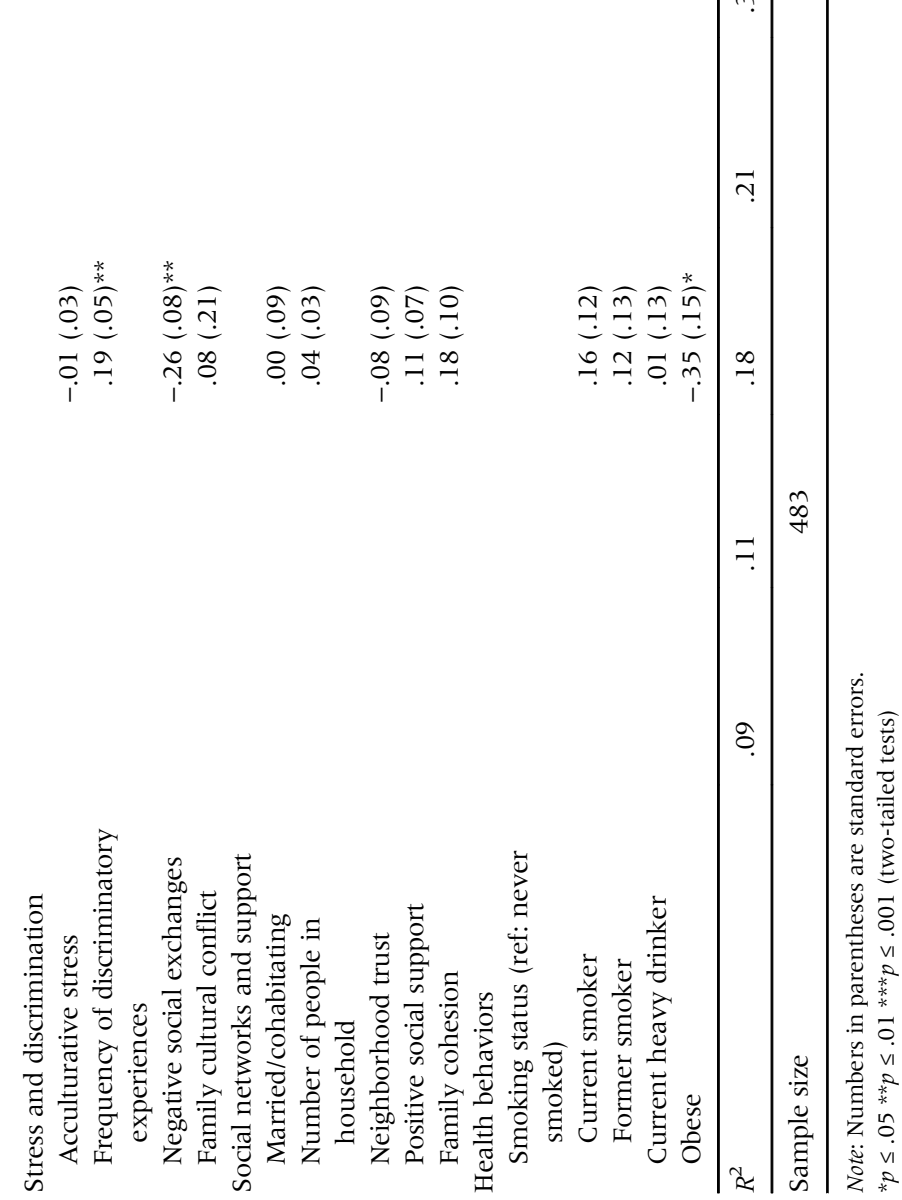

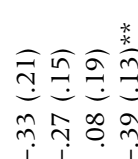

萑

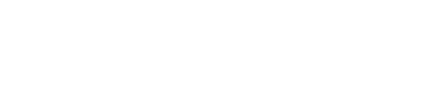

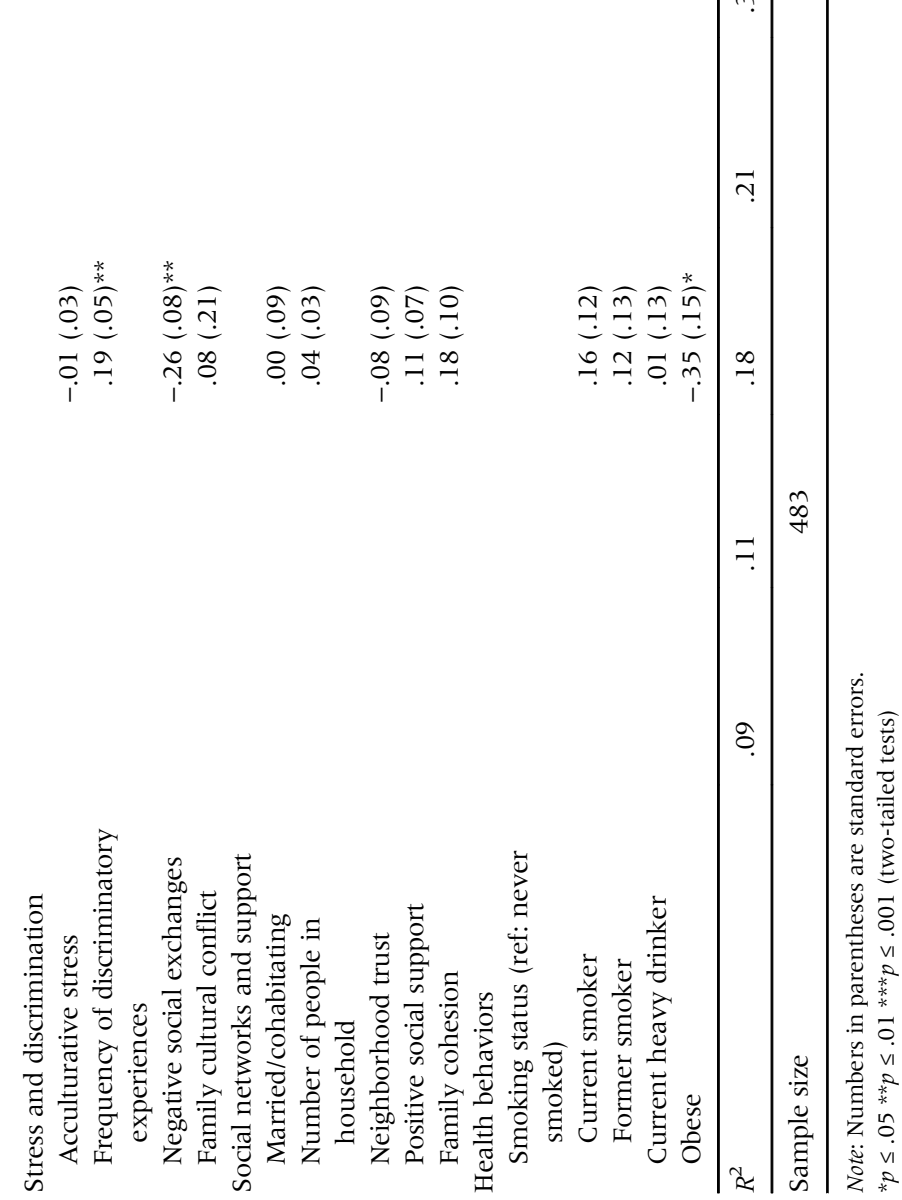

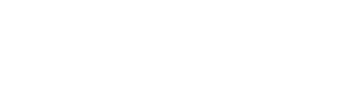


include age, gender, and our acculturation measures, then adding our SES measures, and finally adding our additional mediating measures, including stress and discrimination, social networks and support, and health behaviors. Starting first with the Asian immigrant models, note that one of the most common measures of acculturation-lifecourse timing of migration-is not a significant predictor of self-rated health across any of the Asian immigrant groups. Additional bivariate models (not shown) demonstrate that migrating as a child is positively associated with higher selfrated health for Chinese and Vietnamese, but not Filipino immigrants. However, this significant association is mediated by the other acculturation measures that we utilize in our full models.

Next, we see a broadly similar pattern across groups for the language measures, where compared to bilingual immigrants, those who are Asian-language dominant-typically a marker of lower acculturation-report lower self-rated health. With the exception of Vietnamese immigrants, there are very few additional associations between the acculturation measures and self-rated health. Chinese immigrants who use English at home more frequently report slightly better self-rated health. For Vietnamese immigrants, remitting money to relatives and being a U.S. citizen are associated with significantly better self-rated health, although both associations are partially mediated by the inclusion of the SES measures, specifically relative income for citizenship (using a Sobel test for mediation, $Z=3.51, p<.001$ ) and not having enough money to meet needs $(Z=2.21, p>.05)$, and for remitting, not having enough money to meet needs $(Z=2.75, p<.01)$, indicating that part of the beneficial influence of remitting is due to higher SES.

Finally, in Model 3 for each group we add our remaining mediator variables. Across all three ethnic groups, with the exception of the remitting and citizenship associations for Vietnamese immigrants, we see very little evidence that any of our proposed mediators influence the association between acculturation and self-rated health, which is unsurprising given that only a few of the acculturation measures are meaningful predictors of self-rated health. This is particularly clear for the language coefficients, which remain very similar across all three models for all three groups, despite controlling for a wide variety of proposed mechanisms that may link acculturation and health status. Clearly, language proficiency is a key predictor of self-rated health, with bilingual immigrants reporting the best self-rated health across all ethnic groups, although the distinction between bilingual and English-dominant speakers is suggestive, it is not significant.

Table 3 reports the same series of regression results for Latino immigrants, and, unlike for Asian immigrants, we see a significant number of associations between acculturation and self-rated health, with divergent results for the acculturation measures across the three ethnic groups. For Mexicans in Model la, the frequency of English use at home is positively associated with self-rated health, as is the degree of coethnic ties, indicating some potentially contradictory associations between acculturation and self-rated health. For Cubans, relative to being bilingual, those who are Spanish-dominant report lower self-rated health. In addition, remitting money to relatives is positively associated with self-rated health. Puerto Ricans who are Spanish-dominant report much lower levels of self-rated health compared to those who are bilingual. Other than the language measures, acculturation does not seem to play much of a role in predicting self-rated health for Puerto Ricans (except for a significant, negative effect of migrating as a child in Model 1, but this is reduced to nonsignificance once we adjust for SES in Model 2). For all three Latino groups, bivariate models (not shown) indicate significant, positive relationships between migrating as a child and self-rated health, but this relationship is attenuated (or reversed, in the case of Puerto Ricans) with the inclusion of our additional acculturation measures as shown.

Across Models 2a and 3a for Mexican immigrants, there is no significant mediation of the frequency of English use effect with the addition of SES and other mediators. Interestingly, the frequency of discriminatory experiences is positively associated with self-rated health while negative social exchanges are negatively associated with self-rated health. For Cubans we see a slight attenuation of the Spanish-dominant association with self-rated health once we control for SES, attributable in part to remittances $(Z=-2.16, p<.05)$; we also see an attenuation of the association between remitting and self-rated health, but this attenuation did not achieve significance with a Sobel test for mediation. Also of note for Cubans is that negative social exchanges are negatively 
related to self-rated health while number of residents in the household, as well as positive social support, are positively related to self-rated health. The inclusion of these potential mediators, however, does not substantively influence the relationships between acculturation and self-rated health. In addition, after controlling for SES and the rest of the covariates, age at migration emerges as a significant, negative predictor of self-rated health for Cubans such that migrating as a child is negative for health status. Finally, in Model 2c, for Puerto Ricans, we see an attenuation of the influence of Spanish-dominance on self-rated health with the inclusion of the SES measures. The primary mediator of this relationship is having attended any college $(Z=-3.33$, $p<.001)$. Similar to the results for Cubans, the number of people in the household is positively related to health status for Puerto Ricans. After the inclusion of all of our potential mediating variables, Spanish-dominance remains a strong, negative influence on self-rated health for Puerto Ricans.

In order to check the robustness of our results, and because of concerns that the concept of self-rated health could have different meanings to different ethnic groups (or that the question may be interpreted differently by different groups), we tested correlations between self-rated health and objective health measures (i.e., ever had arthritis/rheumatism, chronic back/neck problems, severe headaches, other chronic pain, seasonal allergies, stroke, heart disease, heart attack, high blood pressure, asthma, chronic lung disease, diabetes, ulcer, epilepsy, cancer, and the total number of chronic conditions) in our data for each group (not shown). Correlations between self-rated health and objective health measures were in the same direction across ethnic groups, without exception, although the magnitude of the correlation varied. No group, however, stood out as having particularly high or low correlations between health conditions and self-rated health. We also tested three objective, diagnosed health measures as outcomes-hypertension, diabetes, and heart disease-to see whether the relationship between our acculturation measures and these outcomes differed from those for self-rated health (not shown). In general, observed acculturation relationships for self-rated health were repeated in models for the objective conditions, although it varied by outcome. In addition, we tested whether self-rated health responses varied systematically by language of interview for our bilingual respondents (who might have chosen to take the survey in either English or their native language). Although our sample sizes in each group were small, we did not find evidence that mean scores on the self-rated health measure varied by language of interview. Thus, we feel confident that there are not major problems with translation issues for the self-rated health question.

\section{Discussion}

Our results highlight the importance of more nuanced analysis of the acculturation-health relationship. We find mixed support for our hypothesis that bilingual immigrants report better self-rated health relative to both native- and English-language dominant immigrants. While native-language dominance consistently has a significant, negative effect on self-rated health (except for Mexicans) even after accounting for measures of SES, stress and discrimination, social networks and social support, and health behaviors, we do not find similar results for Englishlanguage dominance. However, beyond language we find limited evidence of systematic patterns in our acculturation measures that cut across individual ethnic groups. We do find that remitting money home is positive for the health of Vietnamese and Cuban immigrants, which suggests that remittances may mean something different for refugee/asylee groups. Also, strong coethnic ties appear to be beneficial for Mexican immigrants, although the significance of this factor is reduced in the full models. Citizenship emerges as an important predictor of self-rated health only for Vietnamese immigrants. Additionally, we are struck by the lack of significant effects for age at migration (except for Cuban immigrants and for Puerto Ricans), a commonly employed proxy for acculturation status, which has been used in many of the studies demonstrating a negative relationship between acculturation and self-rated health (Lopez-Class et al. 2011). Taken all together, 
our findings suggest that how scholars measure acculturation may strongly influence their findings, and caution against simplistic conclusions that acculturation is good or bad for the health status of immigrants.

First, for all country-of-origin groups but Mexicans, results demonstrate that being bilingualin English and one's native language-is associated with higher self-rated health compared to native-language dominance. This trend holds both for immigrants coming from countries where little English is spoken — such as Cuba and Vietnam — as well as those from more bilingual contexts, like Puerto Rico and the Philippines, where English is an official language. While controlling for SES, along with stress and discrimination and social support measures, does slightly attenuate the differences among the language proficiency groups, bilingualism remains a significant contrast, particularly from those who are native-language dominant. Following the bidimensional model of acculturation articulated earlier, we do not consider bilingualism to be a classification of high or low acculturation. Rather, we are informed by Alejandro Portes and Ruben Rumbault (2001) who have advanced a theory of selective acculturation where immigrants use strong social support networks as protection against discrimination and for assistance with navigating the U.S. educational system and labor market to achieve upward assimilation through bilingualism and biculturalism. This contention is supported by scholarship in psychology finding that strong proficiency in both languages reflects a "cultural flexibility," which is beneficial for psycho-social wellbeing by allowing bilingual immigrants to more easily navigate the culture of sending and receiving countries (Chen, Benet-Martínez, and Bond 2008; Lang et al. 1982; Ying 1995). Altogether, this scholarship suggests that bilingual immigrants may be able to retain social support from coethnics while also having the language skills necessary to advance economically and form ties with nativeborn Americans, and our findings show that these advantages should extend to better self-rated health.

However, our conclusions on the benefits of bilingualism are tempered when we consider that there is no evidence that bilingualism is significantly better than English-language dominance in terms of health status, despite previous research showing a positive relationship between bilingualism and self-rated physical health (Mulvaney-Day, Alegría, and Sribney 2007; Schachter et al. 2012). It is possible that given the importance of English for accessing health care in the United States, English fluency, regardless of native-language maintenance, plays an important role in determining immigrant health status. Nevertheless, we argue that the negative (although not significant) effects of English dominance relative to bilingualism found for all groups but Cubans, are suggestive that bilingualism may be advantageous for immigrant health status across at least some ethnic groups.

To the best of our knowledge, little other scholarship has studied the effects of bilingualismas opposed to just English proficiency-on health, perhaps due to the starkly different results obtained for Mexicans compared to other groups. Interestingly, we do find that higher frequencies of English use at home is positive for the health status of Mexicans, which we interpret as evidence that language is related to the self-rated health of Mexicans, but that the relationship operates somewhat differently compared to other immigrant groups. Indeed, in their analysis of the role of language proficiency in health status, Anthony Jerant, Rose Arellanes, and Peter Franks (2008) found different moderating effects of language for Mexicans compared to other ethnic groups and called for further research that disaggregates Hispanics into country-of-origin groups. Our results support this finding, and highlight a potential trap for scholarship that assumes population samples with high ratios of Mexicans are representative of all Latinos. While this strategy is often employed due to the difficulty of obtaining large enough sample sizes of non-Mexican Latino subgroups, doing so may hide important findings, such as the role of bilingualism in the self-rated health of Cubans and Puerto Ricans. Nevertheless, we recognize that scholars are often confronted with more ethnically limited samples, and in these cases we suggest limiting analysis to the largest single subethnic group. Alternatively, when this strategy is not possible we recommend scholars include indicators of the context of reception, such as legal status and the percent of coethnics in the neighborhood, as rough proxies for the important differences associated with 
country of origin groups, although we caution that this is far from ideal. Similarly, scholarship on Asian immigrant health has also focused on English acquisition (Salant and Lauderdale 2003), perhaps because English proficiency rates are high for many Asian subethnic groups. However, our results indicate that even for immigrants from the Philippines-where English is the dominant language used by the government and in schools-fluency in a traditional, non-English language is positively related to health, and we urge scholars to consider bilingualism in future studies of Asian immigrant outcomes.

Our results also demonstrate that other measures of acculturation have inconsistent effects on self-rated health. First, we find that remitting is positive for the health statuses of Vietnamese and Cuban immigrants, but that it has no significant effect for other immigrant groups. In additional models (not shown), we tested whether there might be nonlinear effects of remittances on self-rated health, and we find that remittances are more strongly associated with self-rated health at the bottom of the scale-fair or poor health-than at the top of the scale-good or excellent health. In other words, remittances are protective of poor health for Vietnamese and Cuban immigrants but do not provide additional benefits at the upper end of the distribution. We speculate that we may find this pattern for Vietnamese and Cuban immigrants but not for other groups because of the unique incorporation experiences of both groups as political asylees/refugees in the United States, as well as conditions in their countries of origin (Portes and Rumbaut 2001; Portes and Zhou 1993). Alternatively, Mariano Sana and Massey (2005) have suggested that measures of remittances may be inaccurate for Mexican immigrants, who are often able to take money with them on trips to Mexico given its proximity to the United States, so it is possible that our measure of remittances does not truly capture the remitting behavior of Mexican (and other) immigrants. All together, more research on the relationships among country of origin, remittance practices, and health status are needed to clarify this finding.

Second, as hypothesized, we find evidence that having a strong ethnic identity as measured by the strength of respondents' ties to coethnics is associated with better self-rated health for Mexican immigrants, but this association is attenuated by the collective effects of our proposed mediators. The ability to account for citizenship among our sample is an important strength of this analysis. Interestingly, however, only for Vietnamese immigrants does U.S. citizenship emerge as a significant predictor of self-rated health. Finally, there are two additional findings worth highlighting. First, despite its near-universal usage in immigrant health disparities research as a primary measure of acculturation, we find no relationship between the duration of time spent in the United States and self-rated health, and we also find that age at migration is only significant for Puerto Ricans and for Cuban immigrants. These findings should further convince scholars of the benefits of including multiple measures of acculturation in their models, which speak to immigrants' social and cultural ties to their countries-of-origin and to the United States. Second, we note that compared to our other, objective measures of SES, relative income is significant in the full additive models much more often. Our finding that traditional SES measures are not important predictors of self-rated health supports other studies finding suppressed effects of SES on health for immigrants (Goldman et al. 2006; Kimbro et al. 2008). Moreover, the fact that relative income-a subjective measure of one's income relative to others-is significant suggests that immigrants view their health status more positively when they believe that they fall more favorably along the socioeconomic spectrum compared to other persons living in the United States (Schnittker and McLeod 2005).

There are some limitations to our study. First, our analysis employs cross-sectional data and we cannot employ longitudinal measures of health status or acculturation. Additionally, although we include multiple measures of acculturation to develop a more nuanced view of immigrants' cultural fluency in their countries-of-origin and the United States, it remains possible that additional, unobserved factors such as media consumption and the adoption of other "Americanized" norms and values may more accurately capture immigrants' levels of acculturation. In particular, while we believe that our measures accurately represent immigrants' ties to their countries-oforigin, our study lacks similar measures focusing on immigrants' ties to the United States, such as 
having a bank account or homeownership. However, we remain confident that our analysis, with its focus on six major U.S. immigrant ethnic groups, significantly expands upon the current norm of relying on only a few measures of acculturation-namely age at migration, duration in the United States, and English proficiency-and represents a significant step forward in the literature examining immigrant health disparities. Furthermore, our results demonstrate that it is inaccurate to conclude that acculturation is necessarily bad for immigrant health outcomes; rather, learning English while maintaining strong fluency in a native language, along with remitting money home and developing a strong ethnic identity, are all associated with better self-rated health, providing further support for bidimensional acculturation models. The process of immigrant adaptation as related to health status, and the fate of the second generation, will have significant impacts on the overall health of the United States in the decades to come, further stressing the importance of more theoretically and empirically nuanced analyses of the relationships among acculturation and health status for immigrant groups.

\section{References}

Abraído-Lanza, Ana, Maria Chao, and Karen Flórez. 2005. “Do Healthy Behaviors Decline with Greater Acculturation?: Implications for the Latino Mortality Paradox." Social Science $\theta$ Medicine 61 (6):1243-55.

Akresh, Ilana Redstone and Reanne Frank. 2011. "At the Intersection of Self and Other: English Language Ability and Immigration Labor Market Outcomes." Social Science Research 40(5):1362-70.

Alba, Richard and Victor Nee. 1997. "Rethinking Assimilation Theory for a New Era of Immigration." International Migration Review 31(4):826-74.

. 2003. Remaking the American Mainstream. Cambridge, MA: Harvard University Press.

$\rightarrow$ Alegria, Margarita, Doryliz Vila, Meghan Woo, Glorisa Canino, David Takeuchi, Mildred Vera, Vivian Febo, Peter Guarnaccia, Sergio Aguilar-Gaxiola, and Patrick Shrout. 2004. "Cultural Relevance and Equivalence in the NLAAS Instrument: Integrating Etic and Emic in the Development of Cross-Cultural Measures for a Psychiatric Epidemiology and Services Study of Latinos." International Journal of Methods in Psychiatric Research 13(4):270-88.

An, Ning, Susan D. Cochran, and Vickie M. Mays. 2008. "Influence of American Acculturation on Cigarette Smoking Behaviors among Asian American Aubpopulations in California." Nicotine $\theta$ Tobacco Research 10(4):579-87.

Angel, Ronald and Peter J. Guarnaccia. 1989. "Mind, Body, and Culture: Somatization among Hispanics." Social Science o Medicine 28(12):1229-38.

Antecol, Heather and Kelly Bedard. 2006. "Unhealthy Assimilation: Why do Immigrants Converge to American Health Status Levels?” Demography 43(2):337-60.

$\rightarrow$ Arnold, Fred. 1989. “Unanswered Questions About the Immigration Multiplier." International Migration Review 23(4):889-92.

$\rightarrow$ Ayers, John W. 2010. "Measuring English Proficiency and Language Preferece: Are Self-Reports Valid?" American Journal of Public Health 100(8):1364-66.

Berry, John. 1997. “Lead Article: Immigration, Acculturation, and Adaptation.” Applied Psychology: An International Review 46(1):5-68.

$\rightarrow$ Bethel, Jeffrey W. and Marc B. Schenker. 2005. "Acculturation and Smoking Patterns among Hispanics A Review." American Journal of Preventive Medicine 29(2):143-48.

Brown, E. Richard, Victoria D. Ojeda, Roberta Wyn, and Rebecca Levan. 2000. “Racial and Ethnic Disparities in Access to Health Insurance and Health Care." Los Angeles, CA: UCLA Center for Health Policy Research.

$\rightarrow$ Bzostek, Sharon, Noreen Goldman, and Anne R. Pebley. 2007. "Why do Hispanics in the USA Report Poor Health?" Social Science $\theta$ Medicine 65(5):990-1003.

$\rightarrow$ Chen, Svlvia X., Verónica Benet-Martínez, and Michael H. Bond. 2008. “Bicultural Identity, Bilingualism, and Psychological Adjustment in Multicultural Societies: Immigration-Based and Globalization-Based Acculturation." Journal of Personality 76(4):803-38.

$\rightarrow$ Chiswick, Barry R. 1999. "Are Immigrants Favorably Self-Selected?" American Economic Review 89(2):181-85. Cho, Yongtae and Robert Hummer. 2001. "Disability Status Differentials across Fifteen Asian and Pacific Islander Groups and the Effect of Nativity and Duration of Residence in the U.S." Social Biology $48(3-4): 171-95$. 
DeSipio, Louis. 2002. “Sending Money Home . . . For Now: Remittances and Immigrant Adaptation in the United States." Pp. 157-87 in Sending Money Home: Hispanic Remittances and Community Development, edited by Rodolfo de la Garza and Briant Lindsay Lowell. Lanham, MD: Rowman \& Littlefield.

$\rightarrow$ Erosheva, Elena, Emily C. Walton, and David T. Takeuchi. 2007. "Self-Rated Health among Foreign- and U.S.-Born Asian Americans: A Test of Comparability." Medical Care 45(1):80-87.

$\rightarrow$ Finch, Brian. K. and William A. Vega. 2003. "Acculturation Stress, Social Support, and Self-Rated Health among Latinos in California." Journal of Immigrant Health 5(3):109-17.

$\rightarrow$ Franks, Peter, Marthe R. Gold, and Kevin Fiscella. 2003. “Sociodemographics, Self-Rated Health, and Mortality in the U.S." Social Science $\theta$ Medicine 56(12):2505-14.

$\rightarrow$ Franzini, Louisa and Maria E. Fernandez-Esquer. 2004. "Socioeconomic, Cultural, and Personal Influences on Health Outcomes in Low Income Mexican-Origin Individuals in Texas." Social Science $\theta$ Medicine 59(8):1629-46.

$\rightarrow$ Frisbie, W. Parker, Youngtae Cho, and Robert A. Hummer. 2001. "Immigration and the Health of Asian and Pacific Islander Adults in the United States." American Journal of Epidemiology 153(4):372-80.

Goldman, Noreen, Rachel T. Kimbro, Cassio M. Turra, and Anne R. Pebley. 2006. "Socioeconomic Gradients in Health for White and Mexican-Origin Populations." American Journal of Public Health 96(12):2186-93.

$\rightarrow$ Gorman, Bridget K., Elaine H. Ecklund, and Holly E. Heard. 2010. "Nativity Differences in Physical Health: The Roles of Emotional Support, Family, and Social Integration. Sociological Spectrum 30(6):671-94.

Grieco, Elizabeth M. 2010. "Race and Hispanic Origin of the Foreign-Born Population in the United States: 2007." American Community Survey Reports. Washington, DC: U.S. Census Bureau.

$\rightarrow$ Heeringa, Steven G., James Wagner, Myriam Torres, Naihua Duan, Terry Adams, and Patricia Berglund. 2004. "Sample Designs and Sampling Methods for the Collaborative Psychiatric Epidemiology Studies (CPES)." International Journal of Methods in Psychiatric Research 13(4):221-40.

Hirschman, Charles and Douglas S. Massey. 2008. “Places and Peoples: The New American Mosaic.” Pp. 1-22 in New Faces in New Places: The Changing Geography of American Immigration, edited by Douglas S. Massey. New York: Russell Sage Foundation.

House, James and David R. Williams. 2000. “Understanding and Reducing Socioeconomic and Racial/Ethnic Disparities in Health." Pp. 81-124 in Promoting Health: Intervention Strategies from Social and Behavioral Research, edited by Brian D. Smedley and S. Leonard Syme. Washington, DC: National Academy Press.

$\rightarrow$ Hunt, Linda M., Suzanne Schneider, and Brendon Comer. 2004. "Should 'Acculturation' be a Variable in Health Research? A Critical Review of Research on U.S. Hispanics." Social Science $\theta$ Medicine 59(5):973-86.

$\rightarrow$ Idler, Ellen L., Louise B. Russell, and Diane Davis. 2000. “Survival, Functional Limitations, and Self-Rated Health in the NHANES I Epidemiologic Follow-Up Study, 1992." American Journal of Epidemiology 152(9):874-83.

$\rightarrow$ Idler, Ellen L. and Ronald J. Angel. 1990. “Self-Rated Health and Mortality in the NHANES-I Epidemiologic Follow-Up Study." American Journal of Public Health 80(4):446-52.

$\rightarrow$ Idler, Ellen L. and Yael Benyamini. 1997. "Self-Rated Health and Mortality: A Review of Twenty-Seven Community Studies." Journal of Health and Social Behavior 38(1):21-37.

Jasso, Guillermina, Douglas S. Massey, Mark R. Rosenzweig, and James P. Smith. 2004. "Immigrant HealthSelectivity and Acculturation." Pp. 227-66 in Critical Perspectives on Racial and Ethnic Differences in Health in Late Life, edited by Norman B. Anderson, Rodolfo A. Bulatao, and Barney Cohen. Washington, DC: The National Academies Press:

$\rightarrow$ Jerant, Anthony, Rose Arellanes, and Peter Franks. 2008. "Health Status among US Hispanics: Ethnic Variation, Nativity, and Language Moderation." Medical Care 46(7):709-17.

$\rightarrow$ Johnson, Kenneth M. and Daniel T. Lichter. 2008. “Natural Increase: A New Source of Population Growth in Emerging Hispanic Destinations in the United States." Population and Development Review 34(2):327-46.

$\rightarrow$ Kandula, Namratha R., Diane S. Lauderdale, and David W. Baker. 2007. "Differences in Self-Reported Health among Asians, Latinos, and Non-Hispanic Whites: The Role of Language and Nativity." Annals of Epidemiology 17(3):191-98.

$\rightarrow$ Kawachi, Ichiro and Lisa F. Berkman. 2000. "Social Ties and Mental Health." Journal of Urban Health $78(3): 458-67$.

$\rightarrow$ Kimbro, Rachel T. 2009. "Acculturation in Context: Gender, Age at Migration, Neighborhood Ethnicity, and Health Behaviors." Social Science Quarterly 90(5):1145-66.

$\rightarrow$ Kimbro, Rachel T., Sharon Bzostek, Noreen Goldman, and Germán Rodriguez. 2008. “Race, Ethnicity, and the Education Gradient in Health." Health Affairs 27(2):361-72.

Kritz, Mary M. and Douglas T. Gurak. 2004. "Immigration and a Changing America." Pp. 259-301 in The American People: Census 2000, edited by Reynolds Farley and John Haaga. New York, Russell Sage Foundation. 
$\rightarrow$ Krueger, Patrick and Virginia Chang. 2008. “Being Poor and Coping with Stress: Health Behaviors and the Risk of Death." American Journal of Public Health 98(5):889-96.

$\rightarrow$ Landale, Nancy S., R. S. Oropesa, and Bridget K. Gorman. 2000. "Migration and Infant Death: Assimilation or Selective Migration among Puerto Ricans?" American Sociological Review 65(6):888-909.

$\rightarrow$ Lang, John G., Ricardo F. Munoz, Guillermo Bernal, and James L. Sorensen. 1982. "Quality of Life and Psychological Well-Being in a Bicultural Latino Community." Hispanic Journal of Behavioral Sciences 4(4):433-50.

$\rightarrow$ Lara, Marielena, Cristina Gamboa, Iya Kahramanian, Leo Morales, and David Hayes Bautista. 2005. “Acculturation and Latino Health in the United States: A Review of the Literature and its Sociopolitical Context." Annual Review of Public Health 26(1):367-97.

Larsen, Luke. J. 2004. "The Foreign-Born Population in the United States: 2003." Current Population Reports. Washington, DC: U.S. Census Bureau.

$\rightarrow$ Lopez-Class, Maria, Felipe González Castro, and Amelie G. Ramirez. 2011. "Conceptions of Acculturation: A Review and Statement of Critical Issues." Social Science $\theta$ Medicine 72(9):1555-62.

Lozano-Ascencio, Fernando. 2005. Remittance Behaviour among Latin American Immigrants in the United States. Presented at the XXV International Union for the Scientific Study of Population International Population Conference, July 21, Tours, France.

$\rightarrow$ Månsson, Nils-Ove and Lennart Råstam. 2001. "Self-Rated Health as a Predictor of Disability Pension and Death-A Prospective Study of Middle-Aged Men." Scandinavian Journal of Public Health 29(2):151-58.

Markides, Kyriakos and Karl Eschbach. 2005. “Aging, Migration, and Mortality: Current Status of Research on the Hispanic Paradox." Journals of Gerontology Series B: Psychological Sciences and Social Sciences 60(Special Issue 2):S68-S75.

Martin, Philip and Elizabeth Midgeley. 2006. "Immigration: Shaping and Reshaping America. Revised and Updated $2^{\text {nd }}$ Edition." Population Bulletin 61(4). Washington, DC: Population Reference Bureau.

$\rightarrow$ Massey, Douglas S. and Ilana R. Akresh. 2006. "Immigrant Intentions and Mobility in a Global Economy: The Attitudes and Behavior of Recently Arrived U.S. Immigrants." Social Science Quarterly 87(5):954-71.

$\rightarrow$ McGee, Daniel L., Youlian Liao, Guichan Cao, and Richard S. Cooper. 1999. “Self-Reported Health Status and Mortality in a Multiethnic U.S. Cohort." American Journal of Epidemiology 149(1):41-46.

Menjívar, Cecilia. 2000. Fragmented Ties: Salvadoran Immigrant Networks in America. Berkeley: University of California Press.

$\rightarrow$ Meredith, Lisa S. and Albert L. Siu. 1995. "Variation and Quality of Self-Report Health Data: Asians and Pacific Islanders Compared with Other Ethnic Groups." Medical Care 33(11):1120-31.

$\rightarrow$ Mossakowski, Krysia N. 2003. “Coping with Percieved Discrimination: Does Ethnic Identity Protect Mental Health?" Journal of Health and Social Behavior 44(3):318-31.

Mulvaney-Day, Norah, Margarita Alegría, and William Sribney. 2007. “Social Cohesion, Social Support, and Health among Latinos in the United States." Social Science $\theta$ Medicine 64(2):477-95.

Palinkas, Lawrence A. and Sheila M. Pickwell. 1995. "Acculturation as a Risk Factor for Chronic Disease among Cambodian Refugees in the United States." Social Science $\theta$ Medicine 40(12):1643-53.

$\rightarrow$ Pennell, Beth-Ellen, Ashley Bowers, Deborah Carr, Stephanie Chardoul, Gina-Qian Cheung, Karl Dinkelmann, Nancy Gebler, Sue Ellen Hansen, Steve Pennell, and Myriam Torres. 2004. “The Development and Implementation of the National Comorbidity Survey Replication, the National Survey of American Life, and the National Latino and Asian American Survey." International Journal of Methods in Psychiatric Research 13(4):241-69.

$\rightarrow$ Phinney, Jean S. 1990. "Ethnic Identity in Adolescents and Adults: Review of Research." Psychological Bulletin 108(3):499-514.

$\rightarrow$ Phinney, Jean S., Gabriel Horenczyk, Karmela Liebkind, and Paul Vedder. 2001. "Ethnic Identity, Immigration, and Well-Being: An Interactional Perspective." Journal of Social Issues 57(3):493-510.

$\rightarrow$ Portes, Alejandro and Min Zhou. 1993. "The New Second Generation: Segmented Assimilation and its Variants." Annals of the American Academy of Political and Social Science 530:74-96.

Portes, Alejandro and Ruben Rumbaut. 2001. Legacies: The Story of the Immigrant Second Generation. Berkeley: University of California Press; and New York: Russell Sage Foundation.

$\rightarrow$ Rogler, Lloyd H. 1994. "International Migrations: A Framework for Directing Research." American Psychologist 49(8):701-08.

Rogler, Lloyd H., Drama E. Cortes, and Robert G. Malgady. 1991. “Acculturation and Mental Health Status among Hispanics: Convergence and New Directions for Research." American Psychologist 46(6):585-97. 
$\rightarrow$ Ryder, Andrew G., Lynn E. Alden, and Delroy L. Paulhus. 2000. “Is Acculturation Unidimensional or Bidimensional? A Head-to-Head Comparison in the Prediction of Personality, Self-Identity, and Adjustment." Journal of Personality and Social Psychology 79(1):49-65.

$\rightarrow$ Salant, Talya and Diane S. Lauderdale. 2003. "Measuring Culture: A Critical Review of Acculturation and Health in Asian Immigrant Populations." Social Science $\theta$ Medicine 57(1):71-90.

$\rightarrow$ Sana, Mariano and Douglas S. Massey. 2005. "Household Composition, Family Migration, and Community Context: Migrant Remittances in Four Countries." Social Science Quarterly 86(2):509-28.

$\rightarrow$ Sanders, Jimy, Victor Nee, and Scott Sernau. 2002. “Asian Immigrants' Reliance on Social Ties in a Multiethnic Labor Market." Social Forces 81(1):281-314.

$\rightarrow$ Schachter, Ariela, Rachel T. Kimbro, and Bridget K. Gorman. 2012. “Language Proficiency and Health Status: Are Bilingual Immigrants Healthier?" Journal of Health and Social Behavior 53(1):124-45.

Schnittker, Jason and Jane McLeod. 2005. "The Social Psychology of Health Disparities." Annual Review of Sociology 31:75-103.

$\rightarrow$ Shetterly, Susan M., Judith Baxter, Lynn D. Mason, and Richard F. Hamman. 1996. "Self-Rated Health among Hispanic versus non-Hispanic White Adults: The San Luis Valley Health and Aging Study." American Journal of Public Health 86(12):1798-1801.

Singer, Audrey. 2004. “The Rise of New Immigrant Gateways.” The Living Cities Census Series. Washington, DC: The Brookings Institution.

$\rightarrow$ Singh, Gopal K. and Mohammad Siahpush. 2002. "Ethnic-Immigrant Differentials in Health Behaviors, Morbidity, and Cause-Specific Mortality in the United States: An Analysis of Two National Data Bases." Human Biology 74(1):83-110.

$\rightarrow$ Thomson, Maria D. and Laurie Hoffman-Goetz. 2009. “Defining and Measuring Acculturation: A Systematic Review of Public Health Studies with Hispanic Populations in the United States." Social Science and Medicine 69(7):983-91.

U.S. Department of Health and Human Services (USDHHS) and the U.S. Department of Agriculture. 2005. Dietary Guidelines for Americans, 2005. 6th ed. Washington, DC: U.S. Government Printing Office.

Vega, William W., Bohdan Kolody, Ramon Valle, and Jude Weir. 1991. “Social Networks, Social Support, and their Relationship to Depression among Immigrant Mexican Women." Human Organization 50(2):154-162.

$\rightarrow$ Vega, William and Hortensia Amaro. 1994. "Latino Outlook: Good Health, Uncertain Prognosis." Annual Review of Public Health 15(1):39-67.

$\rightarrow$ Ward, Louise S. 2010. "Farmworkers at Risk: The Costs of Family Separation." Journal of Immigrant and Minority Health 12:672-77.

Williams, David, Harold W. Neighbors and James S. Jackson. 2008. "Racial/Ethnic Discrimination and Health: Findings from Community Studies." American Journal of Public Health 98(Supplement 1):200-08.

World Health Organization (WHO). 1995. Physical Status: The Use and Interpretation of Anthropometry. WHO Technical Report Series 854. Geneva, Switzerland: World Health Organization.

$\rightarrow$ Ying, Yu-Wen. 1995. "Cultural Orientation and Psychological Well-Being in Chinese Americans." American Journal of Community Psychology 23(6):893-911.

$\rightarrow$ Zhang, Wei and Van M. Ta. 2009. "Social Connections, Immigration-Related Factors, and Self-Rated Physical and Mental Health among Asian Americans." Social Science $\theta$ Medicine 68(12):2104-12.

$\rightarrow$ Zsembik, Barbara A. and Dana Fennell. 2005. "Ethnic Variation in Health and the Determinants of Health among Latinos." Social Science $\theta$ Medicine 61(1):53-63. 